ORIGINAL RESEARCH ARTICLE

\title{
Study on Mesenchymal Artery Syndrome and Its Related Anatomy by MDCTA
}

\author{
Zhaoan $\mathrm{Hu}$,Weiduo Song,Liqiang Liu \\ Medical College, Weifang University of Technology, Shandong, China
}

\begin{abstract}
Objective: To study the effect of MDCTA on the angle and distance of mesenteric artery and abdominal aorta in normal and superior mesenteric artery syndrome and its effect on duodenal level. Materials and Methods: Using MDCTA, retrospective observation of 108 cases of normal and 17 cases of superior mesenteric artery syndrome in patients with superior mesenteric artery and abdominal aortic angle and duodenal level mesenteric artery and abdominal aortic space, and compared. Results: All MDCTA of patients with superior mesenteric artery syndrome showed 'clamp pressure' of the superior mesenteric artery and abdominal aorta in the duodenal segment, causing duodenal obstruction. Among the 108 normal subjects, the angle between the superior mesenteric artery and the abdominal aorta was $46.3^{\circ} \pm 16.5^{\circ}$ and $14.3^{\circ} \pm 5.6^{\circ}$ in the superior mesenteric artery syndrome. The normal level of the duodenum was superior to the superior mesenteric artery Arterial distance of $14.7 \pm 4.4 \mathrm{~mm}$, superior mesenteric artery syndrome in patients with 4.5 $\pm 0.8 \mathrm{~mm}$, the two groups of data have significant statistical differences. Conclusion: MDCTA can show the duodenal obstruction caused by the mesenteric artery and the abdominal aorta of the superior mesenteric artery syndrome, and can accurately show the angle between the superior mesenteric artery and the abdominal aorta the reduction of distance, the diagnosis of superior mesenteric artery syndrome has important clinical value.

KEYWORDS: superior mesenteric artery syndrome; MDCTA; superior mesenteric artery
\end{abstract}

\section{Introduction}

Superior mesenteric artery syndrome (superior mesenteric artery syndrome, SMAS) refers to the duodenal level by the superior mesenteric artery compression caused by acute and chronic obstruction caused by a group of relatively rare clinical syndrome, also known as superior mesenteric artery Compression syndrome, duodenal vascular compression syndrome, benign duodenal stasis, or Wilkie syndrome. Traditionally, both the abdominal aorta and superior mesenteric angiography and duodenal dilatation have been considered to be the most effective diagnostic techniques [1]. However, this method is complicated and expensive, and the patient is subject to more ionization Radiation and other shortcomings. In this study, multi-slice spiral CT angiography (MDCTA) technique was used to measure and compare SMAS and the distance between the superior mesenteric artery (SMA) and the abdominal aorta (AA), and to explore the diagnostic value of MDCTA in SMAS.

\section{Materials and methods}

\subsection{Research objects}

Retrospective extraction in May 2012 to April 13 in Taihe Hospital received upper abdomen MDCTA examination no clear lesions in 78 cases, as a normal control group included in the study, including 48 males and 30 females, the average age of 41.2 years. The use of sequential design method, the extraction of intermittent recurrent postprandial abdominal discomfort discomfort, nausea, vomiting, abdominal pain and other symptoms, clinical suspected mesenteric artery syndrome, parallel gastrointestinal barium meal diagnosis of patients clearly 17 Cases, including 10 males and 7 females, the average age of 43 years; all patients in the digestive tract barium meal diagnosis of a week after the diagnosis of abdominal abdomen MDCTA referral. (See picture 1)

Copyright (C) 2017 -. This is an Open Access article distributed under the terms of the Creative Commons Attribution-NonCommercial 4.0 International License (http://creativecommons.org/licenses/by-nc/4.0/), permitting all non-commercial use, distribution, and reproduction in any medium, provided the original work is properly cited. 
Figure 1. duodenum level of the middle of the spinal column showed a pressure-like pressure, barium through the obstruction, proximal duodenal dilatation, there is a significant reverse peristalsis, notch distal intestinal collapse, barium emptying delay.

\subsection{Check the method}

The scanning device is a 64-row spiral CT scanner (GE, Light Speed 16). Scan parameters: tube voltage 120$140 \mathrm{kV}$, tube current $120 \mathrm{mAs}$, collimator width $16 \times 1.25 \mathrm{~mm}$, pitch pitch $1.375: 1$, scanning time $0.5 \mathrm{~s} / \mathrm{r}$, scanning layer thickness $5 \mathrm{~mm}$. Scanning range from the left diaphragm top to the waist 3 near the level of the use of high-pressure syringes from the elbow median intravenous contrast agent, contrast agent iodine alcohol injection $350 \mathrm{mg} / \mathrm{ml}$, a total of $90-100 \mathrm{ml}$, flow rate $3.5-4 \mathrm{ml} / \mathrm{s}$, Delay time of $20-23 \mathrm{~s}$, are in the suction end breathtaking.

\subsection{Image post-processing}

The reconstructed images are transmitted to the image workstation at the interval of $0.5-1.0 \mathrm{~mm}$ and $1.25 \mathrm{~mm}$ layer thickness respectively. The AW4.2 software is used to reconstruct the MPR Multiple Planar Reconstruction, Volume Rendering, and so on. Observation and measurement of content: normal and SMAS patients with SMA and AA angle; normal and SMAS patients in the duodenal horizontal segment across the abdominal aortic plane to measure the distance between SMA and AA; normal and SMAS patients SMA and The relationship between the angle between the AA and the horizontal segment of the duodenum. All the images were reconstructed by the same radiologist and the corresponding parameters were measured. The subjects were examined by the deputy chief physicians of the two radiologists and evaluated as a result of the agreement. (See Figure 2, Figure 3)

\subsection{Statistical methods}

The results were recorded and sorted. The data obtained were expressed as mean \pm standard deviation $(\mathrm{x} \pm \mathrm{s})$, and SPSS16.0 software was used to carry out group t test. When $\mathrm{P}<0.01$, the difference was statistically significant.

\section{Results}

MDCTA can also show duodenal obstruction and SMA and AA angle and distance reduction. The mean value of SMA and AA in the normal control group was $29.8^{\circ}$ to $62.8^{\circ}$, with an average of $46.3^{\circ}$. The distance between SMA and AA was measured in the duodenal horizontal segment across the AA plane from $10.3 \mathrm{~mm}$ to $19.1 \mathrm{~mm}$, the mean $14.7 \mathrm{~mm}$ for SMAS patients with an angle of $8.7^{\circ}$ to $19.9^{\circ}$ with an angle of $8.7^{\circ}$ to $19.9^{\circ}$ and an average of $14.3^{\circ}$ and a distance of $3.7 \mathrm{~mm}$ to $5.3 \mathrm{~mm}$ between the SMA and AA in the duodenal horizontal segment across the AA plane, The two groups of data through statistical analysis, the $\mathrm{P}$ value of less than 0.01 , with significant statistical differences (see Table 1).

\section{Discussion}

SMAS was originally proposed by Rokitansky in 1861, Wilkie in 1927 for the first time to collect reports of 75 cases of the disease and made a detailed description, then the disease was gradually attracted clinical attention. Despite the controversy, the underlying pathogenesis of SMAS has been generally accepted as a relatively rare disease caused by mechanical and mechanical compression of the duodenum of SMA and AA. The incidence of gastrointestinal barium meal is estimated to be $0.01 \%$ to $0.33 \%$. Clinical manifestations of recurrent postprandial nausea, vomiting, abdominal pain and abdominal distension. Because of the nonspecific and relatively rare symptoms of the disease, many clinicians fail to pay enough attention, resulting in a higher misdiagnosis rate. Domestic literature reported misdiagnosis rate of 
$57.1 \%-67.3 \%$ range. Many of the diseased factors can lead to the depletion of mesenteric and peritoneal fat, leading to the occurrence of SMAS, including chronic wasting diseases (such as: burns, cancer, endocrine disorders), severe trauma (head or spine trauma, The application of gypsum), eating disorders (anorexia nervosa, dyspepsia disease), scoliosis orthopedic surgery or abdominal surgery, can also be due to the exogenous oppression of the tumor, Cui's ligament abnormal high too fixed or mesenteric Arterial low origin.

Table 1. SMAS and normal control group SMA and AA angle and distance CTA measurement results comparison

\begin{tabular}{|c|c|c|c|l|}
\hline & $\begin{array}{c}\text { SMAS group (n } \\
=17)\end{array}$ & $\begin{array}{c}\text { Control group (n } \\
=108)\end{array}$ & t value & Pvalue \\
\hline The angle between & $14.30 \pm 5.60$ & $46.30 \pm 16.50$ & -7.901 & $<0.01$ \\
SMA and AA & & & & \\
\hline SMA and AA distance & $4.5 \pm 0.8 \mathrm{~mm}$ & $14.7 \pm 4.4 \mathrm{~mm}$ & -9.502 & $<0.01$ \\
\hline
\end{tabular}

SMAS diagnostic criteria: the intermittent recurrent postprandial abdominal discomfort discomfort, nausea, vomiting, abdominal pain, symptoms may vary with the body position, weight loss and other clinical manifestations of patients should consider the disease. Typical symptoms combined with upper gastrointestinal imaging and other imaging studies to determine the SMA and AA on duodenal compression caused by obstruction can be diagnosed. But must be excluded can cause duodenal obstruction of other diseases, such as intestinal tumors, intestinal tuberculosis, inflammatory bowel disease, ring pancreas, congenital giant duodenum, intussusception and so on. For patients with atypical clinical manifestations, its non-specific symptoms and chronic gastritis, upper gastrointestinal ulcers, chronic cholecystitis and other common diseases difficult to identify. For repeated treatment of these diseases should not be taken into account the disease, and should be further line imaging examination clear.

Upper gastrointestinal barium meal angiography as a traditional imaging method, in the diagnosis of SMAS occupies a very important position, it can observe the stomach and duodenal mucosa, but also to observe the stomach and duodenum Shape and function and its relationship with adjacent organs. SMAS typical upper gastrointestinal barium meal performance: duodenal level can be seen neatly similar to the pencil compression oblique line, called 'pen' or 'knife cut'. Barium discharge through this slow or even stop, 2-4h cannot emptying; gastric and duodenal obstruction proximal significant expansion, and repeated strong reverse peristalsis or obvious creeping alternating alternating 'bell-like 'Exercise signs, barium can be refluxed into the stomach; hand down in the umbilical push back to the small mesenteric elevation or left lateral position, prone position and chest and knee can ease the oppression of the barium through. However, these manifestations are not specific and can be seen in other diseases such as scleroderma, lupus, small bowel obstruction, pancreatitis, peptic ulcer disease and diabetes. Therefore, mesenteric angiography and low duodenum angiography has been considered the most effective and reliable diagnostic technique. However, this method has the advantages of complex operation, expensive, and the patient needs to bear more ionizing radiation and other shortcomings.

Abdominal MDCTA examination, through a variety of post-processing technology, clearly shows the expansion of the stomach and duodenum intestine at the same time, can observe the angle between SMA and AA, and can clear the SMA for duodenal oppression, while excluding other lesions. MDCTA check non-invasive, fast, simple operation, repeatability, is an ideal check method. Konen et al [10] that, for the diagnosis of SMAS, MDCTA can achieve and traditional DSA angiography similar effect.

In this study, we used MDCTA technique to simultaneously observe the obstruction of the duodenal segment and the expansion of the proximal end of the obstruction, as well as the reduction of the angle and distance between SMA and AA. The mean control group SMA and AA angle of $29.8^{\circ}$ to $62.8^{\circ}$, the average is $46.3^{\circ}$, in the duodenal horizontal segment across the abdominal aortic plane to measure the distance between SMA and AA $10.3 \mathrm{~mm}$ to $19.1 \mathrm{Mm}$, the average is $14.7 \mathrm{~mm}$, which is the same as the use of MDCTA measured by the MDCTA numerical results are the same; the SMAS patients with SMA and AA angle of $8.7^{\circ}$ to $19.9^{\circ}$, the average is $14.3^{\circ}$ The distance between SMA and AA was $3.7 \mathrm{~mm}$ to $5.3 \mathrm{~mm}$, with an average of $4.5 \mathrm{~mm}$ across the abdominal aorta plane in the duodenal horizontal segment, and in the literature [12] published by Unal B using ultrasound and CT The numerical results of patients with SMAS 
were also consistent. We will MDCTA diagnostic SMAS standard is defined as: through the fault and reconstruction of the image can be found SMA and AA on the duodenal horizontal segment of the 'clamp' and the resulting proximal expansion of obstruction, SMA and AA angle is less than 22 degrees, spacing less than 8 to $11 \mathrm{~mm}$. Accurate numerical setup requires further case accumulation in the future, increasing the sample size to increase the sensitivity and specificity of the defined values.

It should be noted that, in addition to making a diagnosis of SMAS, MDCTA can also find complications that may require immediate surgery, including gastric wall disease and portal vein gas, other complications include SMA and AA angle caused by the left Renal vein compression, the so-called nutcracker effect [17].

Figure 2. SMAS patients, in the transverse position of the duodenum horizontal segment across the abdominal aortic plane to measure the distance between SMA and AA. Figure 3 SMAS patients, using MPR reconstruction, in the sagittal reconstruction of the image to measure the angle between SMA and AA.

\section{Conclusion}

SMAS is a relatively rare disease that often requires rapid diagnosis and treatment. For SMAS suspicious cases, MDCTA is a fast, noninvasive, reliable 'one-stop' check method, you can show SMA and AA 'clamp pressure' duodenal level caused by duodenal obstruction, but also accurate display SMAS patients with SMA and AA angle and distance reduction.

\section{References}

1. Gustafsson L, Falk A, Lukes PJ, et al. Diagnosis and treatment of superior mesenteric artery syndrome. Br J Surg. 1984; 71: 499-501.

2. Lei Zhisheng. Department of superior mesenteric artery syndrome misdiagnosis of eight cases. Clinical errors Diagnosis and Treatment, 1998, 11 (5): 289.

3. Konen E, Amitai M, Apter S, et al. CT angiography of superior mesenteric artery syndrome. AJR. 1998; 171: 1279-1281.

4. Chen Zhixin, Zhang Hanguo, et al. Anatomical study of the mesenteric artery angle. Journal of Practical Radiology, 2005,121 (2): 150-151.

5. Unal B, Aktas A, Kemal G, et al. Superior mesenteric artery syndrome: CT and ultrasonography. Diagn Interv Radiol. 2005; 11: 90-95.

6. Wang Ruitao, Li Binghan, et al. superior mesenteric artery syndrome with nutcracker

7. Syndrome and duodenal diverticulum in 1 case. Journal of the Third Military Medical University, 2011.33 (23): $2507-2511$. 